
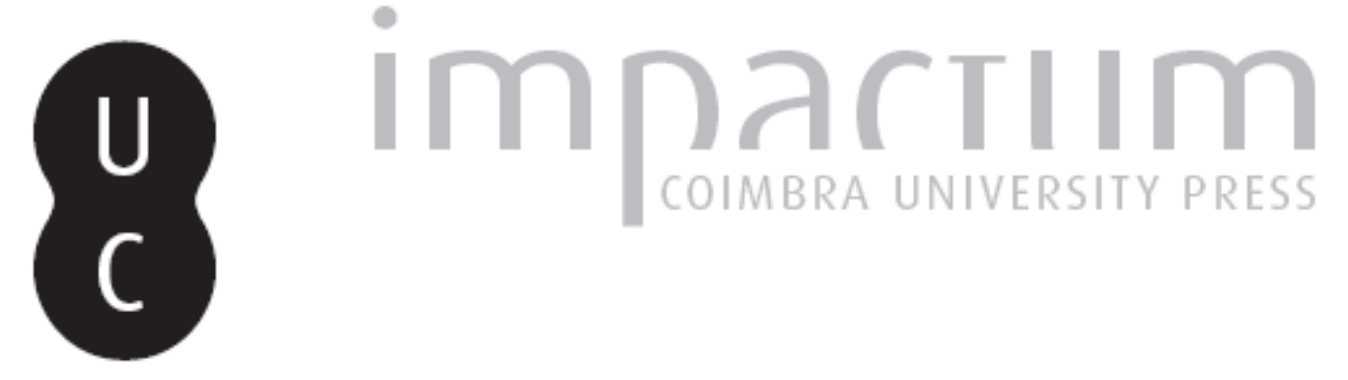

\title{
Dalla scuola romana di Carlo Fontana ai circuiti europei dei Galli Bibiena: architetti italiani in Portogallo nel XVIII secolo
}

\author{
Autor(es): Raggi, Giuseppina
}

Publicado por: Imprensa da Universidade de Coimbra

URL persistente:

URI:http://hdl.handle.net/10316.2/44981

DOI:

DOI:https://doi.org/10.14195/0870-8584_12_18

Accessed : $\quad$ 26-Apr-2023 13:50:30

A navegação consulta e descarregamento dos títulos inseridos nas Bibliotecas Digitais UC Digitalis, UC Pombalina e UC Impactum, pressupõem a aceitação plena e sem reservas dos Termos e Condições de Uso destas Bibliotecas Digitais, disponíveis em https://digitalis.uc.pt/pt-pt/termos.

Conforme exposto nos referidos Termos e Condições de Uso, o descarregamento de títulos de acesso restrito requer uma licença válida de autorização devendo o utilizador aceder ao(s) documento(s) a partir de um endereço de IP da instituição detentora da supramencionada licença.

Ao utilizador é apenas permitido o descarregamento para uso pessoal, pelo que o emprego do(s) título(s) descarregado(s) para outro fim, designadamente comercial, carece de autorização do respetivo autor ou editor da obra.

Na medida em que todas as obras da UC Digitalis se encontram protegidas pelo Código do Direito de Autor e Direitos Conexos e demais legislação aplicável, toda a cópia, parcial ou total, deste documento, nos casos em que é legalmente admitida, deverá conter ou fazer-se acompanhar por este aviso.

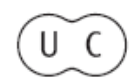




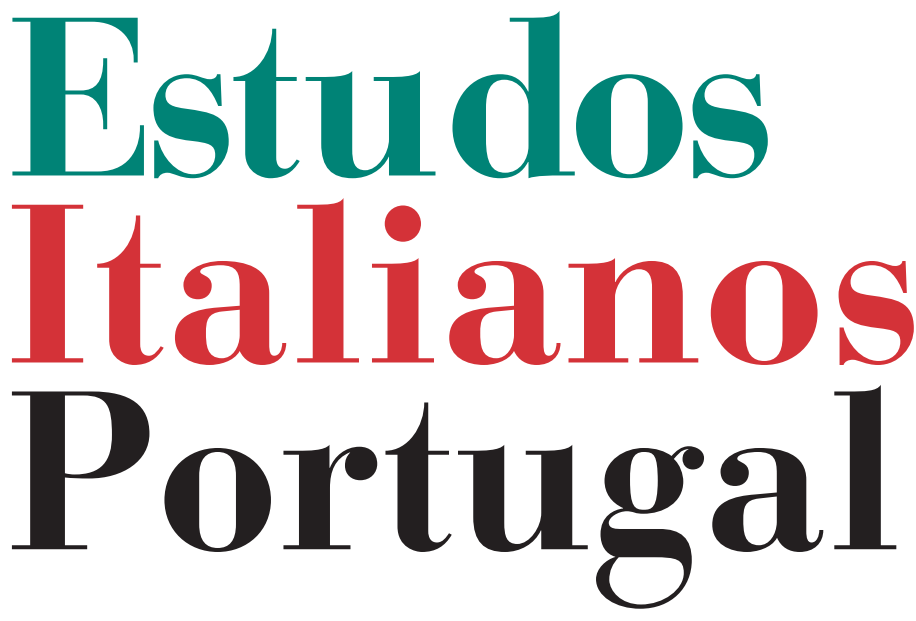

Instituto

Italiano

de Cultura

de Lisboa

Nova Série

No 12

2017 


\title{
DALLA SCUOLA ROMANA DI CARLO FONTANA AI CIRCUITI EUROPEI DEI GALLI BIBIENA: ARCHITETTI ITALIANI IN PORTOGALLO NEL XVIII SECOLO
}

\author{
Giuseppina RaGgi*
}

I RAPPORTI ARTISTICI tra Italia e Portogallo sono sempre stati intensi e ricchi. Nel campo dell'architettura, il Settecento è un periodo particolarmente fecondo grazie ad una serie di circostanze e fattori che è necessario riconsiderare criticamente. Il primo fattore riguarda il ruolo centrale del regno di Giovanni V nel cambio di paradigma dell'architettura e dell'urbanismo lusitani. Egli fu il sovrano che maggiormente interpretò le arti, e soprattutto l'architettura, come lo strumento ideale per esaltare la potenza portoghese nel consesso delle altre monarchie europee. Il secondo fu il terremoto di Lisbona del 1755. Questo evento, paradossalmente e al di là della distruzione, creò le condizioni per ripensare gli innumerevoli progetti delineati anteriormente e per far tesoro dell'esperienza costruttiva maturata nel cantiere di Mafra. Il terzo fu il connubio tra architettura e teatro d'opera, che è un elemento ancora sottostimato dagli studi critici ma centrale per comprendere le dinamiche architettoniche portoghesi. Questa connessione nacque nella prima metà del Settecento in grande parte grazie al ruolo giocato dalla regina Maria Anna d'Asburgo, di origine austriaca, profondamente appas-

\footnotetext{
*Giuseppina Raggi, doutorada em História da Arte pelas Universidades de Bolonha e de Lisboa (2005) e investigadora do Centro de Estudos Sociais da Universidade de Coimbra. Especialista da pintura de quadratura bolonhesa e sua difusão em Portugal e no Brasil, dedica-se atualmente à arquitetura colonial no Brasil e à atividade de Juvarra e dos Bibiena em Portugal. giuseppinaraggi@ces.uc.pt
} 
sionata d'opera ${ }^{1}$. Ciò determinò il progressivo imporsi della cultura architettonica-scenografica di tradizione bolognese, che permeò la cultura portoghese non solo nel XVIII ma anche in gran parte del XIX secolo.

\section{UNA NUOVA VISIONE DELL'ARCHITETTURA E DELLA CITTÀ: FilipPo JuVARRA A LisBona (1719)}

Analizzando l'arrivo in Portogallo di architetti italiani durante il Settecento, è necessario ripensare criticamente il contesto storico-artistico che portò alla chiamata di Filippo Juvarra (Messina, 1678-Madrid, 1736) in Portogallo. Questo evento rappresentò la chiave di volta dello sviluppo settecentesco dell'architettura portoghese. L'architetto siciliano, radicato a Roma e al servizio del re di Savoia dal 1714, soggiornò a Lisbona da gennaio a luglio del $1719^{2}$. Nella storia del regno di Portogallo di Antico Regime nessun altro architetto o artista, straniero o nazionale, venne accolto con uguale entusiasmo né venne incaricato di un piano urbanistico-architettonico di uguale portata. Ciò dimostra l'unicità della temperie culturale che percorse la corte lusitana tra il secondo e il terzo decennio del Settecento, all'interno della quale il soggiorno di Juvarra svolse la doppia funzione di catalizzatore e di climax.

La chiamata dell'architetto italiano da parte del monarca lusitano rappresentò, infatti, l'apice di un movimento di apertura e aggiornamento verso il linguaggio artistico europeo che caratterizzò i primi venti anni del regno di Giovanni $\mathrm{V}$, cioè tra il 1708 e il 1728-1730, e che si tradusse in una

${ }^{1}$ Giuseppina Raggi, "Una lunga passione per l'opera in Portogallo: la reginaconsorte Maria Anna d'Asburgo, l'arte dei Galli Bibiena e nuovi disegni per il Real Teatro dell'Ópera do Tejo", Sabine Frommel; Micaela Antonucci (coord.), Da Bologna all'Europa. Artisti bolognesi in Portogallo (XVI-XIX secolo), Bologna, Bononia University Press, 2017, pp. 159-188.

${ }^{2}$ Vedi bibliografia dell'articolo di Walter Rossa in questo dossier. Per uno sguardo panoramico, seppur datato, vedi Emilio Lavagnino, Gli artisti italiani in Portogallo, Roma, Libreria dello Stato, 1940. 
straordinaria effervescenza progettuale anche in campo profano. Il viaggio europeo che il giovane monarca aveva pianificato in tutti i particolari e che voleva realizzare in incognito durante il 1715 e il 1716 corrispondeva alla sua curiosità e volontà di aggiornamento. Per lo meno sino alla fine degli anni Venti, la politica architettonica di Giovanni V non si focalizzò solo sulla patriarcale e sul complesso di Mafra, anzi, motivò progetti ben più ampi ed ambiziosi inerenti alla nuova Lisbona Occidentale, istituita a seguito della divisione della diocesi della città in funzione della nuova patriarcale nel 1716. Grazie alla sua capacità scenografico-architettonica e prospettico-urbanistica, Filippo Juvarra fu il traduttore più adeguato per dare concreta veste progettuale alla città che venne concepita secondo parametri nuovi, su scala urbanistica e scenografica, con architetture magniloquenti e funzionali appropriate alla grandezza della monarchia e dell'impero che si voleva esaltare.

Nuovi documenti custoditi presso l'Archivio di Stato di Vienna permettono di ripensare in profondità questo perio$\mathrm{do}^{3}$. Filippo Juvarra venne chiamato a Lisbona per ideare e per scegliere la migliore localizzazione di "una Basilica con Palazzo Patriarcale, di un Palazzo Reggio, di una Casa di Campo Reggia, di un Arsenale e altri edifitij”“. Del complesso palatino-patriarcale vennero stesi due progetti: il primo si estendeva nell'area della Ribeira, mentre il secondo era localizzato nell'area di Buenos Aires. L'architetto italiano ideò anche assi viarie lungo il Tago che agevolassero lo spostamento via terra, così come tracciò un più rapido collegamento con Mafra, dove João Frederico Ludovice aveva intrapreso la progettazione della basilica, palazzo e convento. Larchitetto siciliano intervenne in questa fase iniziale del complesso,

\footnotetext{
${ }^{3}$ Giuseppina Raggi, "Filippo Juvarra in Portogallo: documenti inediti per i progetti di Lisbona e Mafra”, ArcHistoR, 7, 2017, http://pkp.unirc.it/ojs/index.php/archistor (consultato a 30-04-2017).

${ }^{4} \mathrm{Ib}$., doc. 3 .
} 
dato che visitò il cantiere di Mafra appena giunto a Lisbona, dando "ad intendere [che] disapprova molto quell'edificio, tanto per rispetto al terreno aspro, deserto, montuoso e senz'acqua, quanto per l'ordine dell'architettura malinteso, e peggio collocato"s.

Fu a partire da ciò che vide a Mafra e dall'elaborazione del secondo progetto per Buenos Aires che si fece urgente ripensare l'organizzazione dei cantieri e l'impiego di maestranze specializzate ${ }^{6}$. Grazie ai tanti "discorsi di Architettura" che il re intratteneva con Juvarra, a palazzo o mentre visitavano la città insieme ai principali esponenti della corte, si configurò un modo nuovo di pensare l'architettura. Tutto ciò avvenne alla presenza di coloro che avrebbero avuto un ruolo fondamentale negli anni successivi: João Frederico Ludovice in primis, ma anche il giovane ingegnere militare Manuel da Maia, che nel 1716 aveva tracciato la mappa di Lisbona con la nuova divisione in Occidentale e Orientale, e che nel 1755 coordinò la ricostruzione della città .

Questa intensa attività progettuale procedette di pari passo con la spinta di novità introdotta alla corte portoghese

\footnotetext{
${ }^{5}$ Ib., doc. 1 .

${ }^{6} \mathrm{Ib}$, doc. 9, 28-02-1719: "Memoria per Milano" in cui si chiedono "cento Mastri Muratori con suoi garzoni per servirli [...] che si potrebbero dividere in num. ${ }^{\text {ro }}$ di 4 o 5 squadre, le quali havessero il suo capo di vaglia, e soprattutto si raccomanda la perfettione de soprad. ${ }^{\text {ti }}$ operarij e la qualità. In ugual num. ${ }^{\text {ro }}$ Scarpellini, o vero piccapietre, li quali siano capaci di fare qualsivoglia scorniciatura o sacome, o modine, e in detti un numero sufficiente almeno di 24 Maestri, che sappiano intagliare li ornamenti, che richiede l'istessa Architettura, e che d. ${ }^{i}$ Maestri siano esperimentati per la sufficienza di poter condurre una grande opera, potrebbono essere parimenti divisi in squadre con $\mathrm{i}$ suoi capi perfetti per dirigere gli altri”.

7 Walter Rossa, "No 1. ${ }^{\circ}$ plano", Ana Tostôes; Walter Rossa (coord.), Lisboa 1758. O plano da Baixa hoje, Lisboa, Câmara Municipal de Lisboa, 2008, pp. 24-81; id., "L’anello mancante: Juvarra sogno e realtà di un'urbanistica delle capitali nella Lisbona settecentesca”, Elisabeth Kieven; Cristina Ruggero (coord.), Filippo Juvarra (16781736). Architetto dei Savoia, architetto in Europa, Roma, Campisano, 2014, vol. 2, pp. 183-196, versione port. id., Fomos condanados à cidade. Uma década de estudos sobre património urbanístico, Coimbra, Imprensa da Universidade de Coimbra, 2015, pp. 313-336.
} 
dall'arrivo, nel 1708, dell'arciduchessa austriaca Maria Anna d'Asburgo come regina di Portogallo. La sovrana rinnovò profondamente la vita di corte, introducendovi il gusto per la musica italiana, principalmente per l'opera ${ }^{8}$. Grazie alla convergenza del rinnovamento culturale promosso da Giovanni V e Maria Anna d'Asburgo, durante il secondo e terzo decennio del Settecento, le nuove visioni in campo architettonico e musicale (inteso anche come scenografia e architettura teatrale) produssero un impatto capace di plasmare l'originalità della vicenda architettonica portoghese durante il XVIII secolo.

\section{TRA CONTINUITÀ E DIVERGENZE:}

Antonio Canevari a Lisbona (1727-1732)

Nel 1727 la chiamata dell'architetto romano Antonio Canevari (Roma, 1681-Napoli, 1764) si inserì in questa linea di continuità, dato che anche lui era stato, come Filippo Juvarra, allievo di Carlo Fontana (1638-1714) ${ }^{9}$. Il contesto politico stava cambiando a causa delle crescenti tensioni diplomatiche tra il regno di Portogallo e la Santa Sede, ma la cultura dell'Arcadia e il linguaggio architettonico romano continuavano ad essere condivisi tra Roma e Lisbona. L'ingaggio di Canevari a servizio della corte lusitana fu motivato dall'imminenza del doppio matrimonio tra le case regnanti di Braganza e di Borbone. Com'è noto l'architetto era stato scelto per progettare a Roma la sede dell'Arcadia, il Bosco

${ }^{8}$ Giuseppina Raggi, "Una lunga passione per l'opera in Portogallo"; id., "Filippo Juvarra a Lisbona: due progetti per un teatro regio e una questione musicale", Filippo Juvarra. Architetto dei Savoia, architetto in Europa, vol 2, pp. 209-228, versione port. ampliata "A idealização de dois projectos para o teatro régio e um novo desenho do arquitecto Filippo Juvarra para a corte portugues", Revista de História da Arte, 11, 2014, pp. 136-151.

9 Sandra Vasco Rocca; Gabriele Borghini (org.), Giovanni V di Portogallo (1707-1750) e la cultura romana del suo tempo, Roma, Àrgos, 1995; Paola Ferraris, "Antonio Canevari a Lisbona (1727-1732)”, ib., pp. 57-66. 
Parrasio, che venne finanziato da Giovanni V e inaugurato nel settembre 1726 non ancora concluso ${ }^{10}$.

Il trasferimento a Lisbona urgeva e l'architetto vi giunse nell'aprile del 1727. Pur non ricevendo le stesse entusiastiche attenzioni dedicate da Giovanni V a Filippo Juvarra, Antonio Canevari venne accolto "favorevolmente" dal monarca "tre giorni dopo il suo arrivo, avendogli fatto somministrare sessanta doppie a titolo di provedersi de mobili che gli sono necessari" ${ }^{11}$. Il coinvolgimento diretto di Canevari nei preparativi e nei festeggiamenti per il doppio matrimonio è documentato. Egli progettò l'edificio effimero costruito sul fiume Caia per lo 'scambio delle principesse' e la macchina del fuoco di artifizio nel Terreiro do Paço in onore del matrimonio tra Barbara di Braganza e il principe Ferdinando di Borbone ${ }^{12}$. A lui può dunque essere ascritta l'invenzione della seconda macchina di fuochi d'artificio, eretta poche settimane dopo per festeggiare l'arrivo a Lisbona della principessa Mariana Vitoria di Borbone, giovane sposa del principe Giovanni. In questa occasione fu costruito un ponte effimero a Belém. La descrizione di quest'opera in Fasto de Hymeneo e la sua riproposizione grafica nella famosa serie di azulejos nel chiostro della chiesa dei Terziari francescani a Salvador da Bahia permette di attribuire a Canevari l'ideazione dell' architettura effimera, così come era accaduto per quella sul fiume Caia, dove era stato presente insieme a João Frederico Ludovice.

La relazione con Ludovice si configura simile a quella stabilita con Filippo Juvarra ${ }^{13}$, dato che in occasione del rifacimento della torre dell'Università di Coimbra il progetto

\footnotetext{
${ }^{10}$ Paola Ferraris, "Il Bosco Parrasio dell'Arcadia (1721-1726)”, ib., pp. 136-151.

${ }^{11}$ Roma, Archivio Segreto Vaticano [ASV], Segr. Stato 84, c. 272, 16-10-1727.

${ }^{12}$ João Castelo-Branco Pereira; Ana Paula Rebelo Correia, Arte efémera em Portugal, Lisboa, Fundação Calouste Gulbenkian, 2000.

${ }^{13}$ Giuseppina Raggi, "Filippo Juvarra in Portogallo: documenti inediti per i progetti di Lisbona e Mafra”.
} 
di Gaspar Ferreira inviato a Lisbona venne criticato "dagli architetti", sottintendendo con ogni probabilita Ludovice e Canevari, ma a quest'ultimo venne richiesto di fornire un nuovo progetto, poi effettivamente realizzato. Canevari costruì anche la torre dell'orologio del palazzo reale della $R i$ beira, dedicandosi alle trasformazioni interne del complesso reale necessarie per accogliere la coppia degli eredi al trono.

Non sono noti, al momento, dati che possano testimoniare il grado di relazione tra il suo intervento e la risistemazione dell'intera area progettata da Juvarra, i cui disegni integravano le collezioni reali. Senza dubbio Canevari era in grado di entrare in sintonia con quanto immaginato negli anni precedenti. A lui si potrebbe attribuire anche il "vago teatrino fatto fare espressamente a tale effetto" ${ }^{14}$ per la rappresentazione a palazzo dell'opera in musica Don Chisciotte nel 1728, così come la ripresa di tracciati urbanistici di Juvarra nel percorso ideato per lo sbarco della principessa Mariana Vitoria da Belém sino al palazzo della Ribeira nel 1729. Le due torri costruite, rispettivamente, nel palazzo reale e all'Università di Coimbra, così come il probabile apporto all'innalzamento di quelle della basilica di Mafra restituiscono la cifra del suo lessico, di cui rimane segno tangibile in Sant'Antão do Tojal, residenza estiva del patriarca di Lisbona Thomás de Almeida ${ }^{15}$.

In questo piccolo nucleo urbano sono sintetizzate le potenziali applicazioni dell'abilità architettonica di Antonio Canevari, tra cui risalta l'aspetto tecnico e scenografico legato al trasporto e alla distribuzione dell'acqua, dato che vi costruì anche l'acquedotto e la fontana monumentale prospiciente alla piazza. Per quanto riguardava Lisbona occidentale, il problema della mancanza d'acqua era sorto evidente durante l'elaborazione del progetto per il sito di Buenos Aires realizzato da Juvarra nel 1719, il quale congetturò sulla necessità di fornire acqua a questa parte della città. Nel 1728 Giovanni

\footnotetext{
${ }^{14}$ Roma, ASV, Segr. Stato 85, c. 57, 10-02-1728.

${ }^{15}$ Paola Ferraris, "Antonio Canevari a Lisbona (1727-1732)".
} 
$\mathrm{V}$ decise di intraprendere la costruzione dell'acquedotto das Águas Livres, affidandolo inizialmente ad Antonio Caneva$\mathrm{ri}^{16}$. La pianificazione di quest'opera diede inizio a un'aspra diatriba con gli ingegneri portoghesi che portò l'architetto romano a chiedere di essere dispensato dal servizio reale nel $1732^{17}$. Lo scontro rivela aspetti interessanti se analizzato a partire dalle diverse prospettive da cui si originò: da una parte la prassi ingegneristica lusitana, dall'altra il modello degli acquedotti romani declinato sul nuovo stile che si stava imponendo a Roma attraverso gli architetti attivi negli anni Venti quali Francesco de Sanctis, Filippo Raguzzini e lo stesso Canevari. Non va neppure dimenticato che Carlo Fontana scrisse l'Utilissimo trattato delle acque correnti (1696) e che Nicola Salvi, principale allievo di Antonio Canevari, iniziò a costruire nel 1731-1732 la fontana di Trevi, addossata a palazzo Poli in posizione similare a quella progettata a Tojal, fatta salva la diversità di scala e di composizione.

Partendo da questa prospettiva d'analisi, il ritorno a Roma di Antonio Canevari supera l'ambito della diatriba ingegneri portoghesi versus architetti italiani, ma denuncia un cambiamento significativo nella politica artistico-culturale di Giovanni V da inquadrare nel contesto politico-diplomatico di quegli anni. Giovanni V smise di spettacolarizzare la potenza della monarchia portoghese sul gran teatro di città di Roma e la protezione del monarca su Antonio Canevari fu di gran lunga inferiore al favore di cui beneficiò Filippo Juvarra, mentre gli ingegneri e architetti militari portoghesi acquisivano progressivamente uno spazio che, in precedenza, era stato riconosciuto esclusivo appannaggio di Juvarra come progettista e di Ludovice come architetto dei cantieri. Le dif-

${ }^{16}$ Irisalva Moita (org.), D. João Ve o abastecimento de água, Lisboa, Câmara Municipal de Lisboa, 1990; Joaquim Oliveira Caetano, "O Aqueduto das Águas Livres", ib., pp. 293-312.

${ }^{17}$ João Luís Lisboa; Tiago C. P. dos Reis Miranda; Fernanda Olival, Gazetas manuscritas da Biblioteca Pública de Évora, Lisboa, Colibri, vol. 2, p. 132. 
ficoltà e le resistenze con cui Antonio Canevari si dovette confrontare, sino alla decisione di chiedere la dispensa del re per tornare in Italia, testimoniano tre fattori importanti:

1) il cambiamento delle priorità politico-culturali di Giovanni $\mathrm{V}$ in concomitanza con la rottura delle relazioni diplomatiche con Roma tra 1728 e 1732;

2) l'acquisizione di una nuova visione da parte degli ingegneri militari portoghesi a partire dal soggiorno in Portogallo di Filippo Juvarra nel 1719, di cui Manuel da Maia rappresenta l'anello di congiunzione;

3) la loro affermazione anche nell'ambito dell'architettura aulica, rispondendo alle nuove committenze di Giovanni V degli anni Trenta e Quaranta a discapito della chiamata di architetti stranieri, in particolare italiani.

Così, a partire dal rientro a Roma di Antonio Canevari, gli unici architetti stranieri che continuarono ad operare in Portogallo durante il regno di Giovanni V non furono più personalità di spicco nella Roma e nell'Europa del tempo, ma figure di più modesto profilo che si naturalizzarono portoghesi, integrando in molti casi i quadri militari.

L'OSMOSI TRA QUADRATURA, SCENOGRAFIA E ARCHITETTURA: Niccolò Nasoni a Oporto (1725-1773)

La peculiare traiettoria professionale di Niccolò Nasoni (San Giovanni Valdarno, 1691-Oporto, 1773) fuoriesce dal quadro appena tracciato per Lisbona e anticipa la via di maggior successo attraverso cui gli architetti italiani continuarono ad alimentare lo sviluppo dell'architettura settecentesca in Portogallo ${ }^{18}$. Sono note le peripezie della sua biografia: nacque a San Giovanni Valdarno e fu scenografo dell'Accademia dei Rozzi di Siena, dove nel 1715 progettò l'arco trionfale

${ }^{18}$ Giuseppina Raggi, "La scenografia come architettura: Niccolò Nasoni e la torre dos Clérigos”, Estudos Italianos em Portugal, n. s., 8, 2013, pp. 125-138, con bibliografia precedente e in particolare gli studi di Giovanni Battista Tedesco. 
innalzato per celebrare l'entrata del nuovo vescovo Alessandro Zondadari. Grazie al successo ottenuto venne inviato a Bologna a perfezionarsi con Stefano Orlandi, quadraturista e scenografo. La protezione dei Zondadari gli propiziò anche la chiamata a servizio dell'Ordine di Malta in occasione dell'elezione di Marcantonio Zondadari a gran maestro nel 1720. Impegnato a realizzare opere di quadratura, Nasoni rimase a La Valletta dal 1722-1723 al 1725. L'improvvisa morte del gran maestro aveva portato nel frattempo all'elezione del portoghese António Manuel de Vilhena, il cui vice-cancelliere era Roque de Távora e Noronha, fratello di Jerónimo de Távora Noronha Leme de Cernache, decano della cattedrale di Oporto che rimase sede vacante dal 1717 al 1740. Fu Roque de Távora e Noronha a invitare Nasoni a Oporto, nella cui cattedrale l'artista lavorava come quadraturista già dal novembre 1725 .

Le connessioni di questi fatti con l'effervescenza progettuale che si viveva in quegli stessi anni a Lisbona sono strette. Thomás de Almeida e Noronha, elevato a primo patriarca della città occidentale nel 1717, era stato vescovo di Oporto dal 1709. Il piano di rinnovamento urbanistico, da lui avviato, venne continuato dal decano Jerónimo de Távora e Noronha. La chiamata di Niccolò Nasoni si inserì in questo contesto, venendogli commissionata la decorazione quadraturistica delle cattedrali di Oporto e Lamego. Il vincolo con la politica artistico-culturale di Giovanni V si rinserrò maggiormente nel momento della rottura delle relazioni diplomatiche con la Santa Sede nel 1728, dato che la nuova situazione politico-diplomatica permise a Nasoni di fare il salto dal campo dell'effimero a quello del costruito, assecondando una possibilità intrinseca alla sua formazione bolognese. Nel 1732 Nasoni venne incaricato di progettare la chiesa di São Pedro dos Clérigos, la cui confraternita era presieduta dallo stesso Jerónimo de Távora e Norona. La scelta di edificare la chiesa rientrava nel piano di riorganizzazione ecclesiastico- 
-diocesana, che incentivava lo sviluppo e il consolidamento delle confraternite dei chierici secolari sia in Portogallo che nei territori ultramarini del Brasile. Questo piano rispondeva alla politica di Giovanni V in campo religioso.

La relazione tra la fondazione della chiesa dos Clérigos di Oporto e l'ordine perentorio del re di portare a termine la basilica di Mafra, nel biennio 1729-1730, viene sancita anche dalla richiesta dell'invio a Oporto nel 1732 della pian$\mathrm{ta}^{19}$. Il progetto originale di Nasoni prevedeva il simbolico impianto ovale accresciuto da due torri campanarie laterali e una cupola. Questi ultimi due elementi, modificati nel tempo per problemi statici, avrebbero conferito all'edificio una chiara risonanza con la basilica di Mafra, rafforzando simbolicamente il confronto politico in atto tra il regno di Portogallo e la Santa Sede. Il dialogo con l'architettura di Mafra non significò, però, la medesima modalità di rapportarsi ai modelli romani. Il chiaro richiamo al lessico di Francesco Borromini evidenzia quanto l'edificio venne interpretato in chiave scenografica. Il 2 giugno 1732 fu posta la prima pietra e la facciata della chiesa venne mostrata grazie all'arte della prospettiva ${ }^{20}$. L'architettura effimera dipinta con quadrature 'edificava' la facciata progettata, stabilendo un nesso evidente tra spazio dipinto e spazio costruito e rivelando la peculiarità del linguaggio di Nasoni.

Nel 1740 la nomina a vescovo di Oporto del francescano José Maria da Fonseca e Évora (1690-1752) continuò a rafforzare la relazione tra la politica del re e la diocesi di Oporto dopo 23 anni di sede vacante. Dal 1712 al 1740 egli aveva vissuto a Roma, assolvendo con successo a diversi incarichi diplomatici. La chiesa dos Clerigos fu consacrata durante il suo vicariato (1741-1752) e la sontuosità degli

${ }^{19}$ B. Xavier Coutinho, A igreja e a Irmandade dos Clérigos. Apontamentos para a sua história, Porto, Câmara Municipal do Porto, 1965, p. 95.

${ }^{20}$ Porto, Arquivo da Igreja dos Clérigos de Porto, Livro de Contas, c. 196 (cit. da Coutinho). 
apparati effimeri allestiti in occasione della sua entrata solenne nel maggio del 1743 portano la firma, anche se non dichiarata esplicitamente nella Relação da solemne entrada pública, dell'abilità costruttivo-scenografica di Niccolò Nasoni. Questa abilità sarà una delle qualità più apprezzate negli architetti di scuola bolognese che, a partire dagli anni Trenta, cercarono spazi di apertura in Portogallo grazie alla passione per l'opera italiana radicata dall'efficace azione della regina Maria Anna d'Asburgo.

TRA TEATRI D'OPERA, RESISTENZE E CONFRONTI:

Roberto Clerici, Niccolò Servandoni e l'assenza 'PRESENTE' DEGLI ARCHITETTI ROMANI (1733 - 1750)

La morte inaspettata del marchese di Abrantes nel 1733 accentuò le nuove opzioni di Giovani V nel campo dell'investimento architettonico. Sin dai tempi dall'ambasciata straordinaria a Roma, il marchese fu un forte sostenitore dell'opera in musica e nei primi anni Trenta si verificò una convergenza di interessi sulla possibilità di aprire un teatro regio pubblico sull'esempio del Nuovo Teatro Regio di Torino progettato da Filippo Juvarra e condotto dai Paghetti ${ }^{21}$. Con la morte del primo marchese Abrantes, l'apertura di un teatro d'opera pubblico ottenne il beneplacito regio nel 1735, grazie all'intermediazione del secondo marchese, ma non il diretto investimento della corona come succedeva nella altri corte europee.

A Lisbona l'attività operistica si estendeva non solo ai teatri pubblici, ma anche in quelli reali montati stabilmente nel palazzo della Ribeira e, forse, nel palazzo reale di Belém. Un fatto pressoché ignorato sino ad ora dagli studi, è la presenza simultanea a Lisbona, tra il 1734 e il 1736, dell'architetto Roberto Clerici (Parma ?-post 1748) e del compositore Gio-

\footnotetext{
${ }^{21}$ Giuseppina Raggi, "Filippo Juvarra a Lisbona: due progetti per un teatro regio e una questione musicale".
} 
vanni Bononcini (Modena, 1670-Vienna, 1747). Roberto Clerici (il giovane) era stato allievo di Ferdinando Bibiena a Bologna da dove "partì pratico disegnatore, e fece studi di teatri, ma poi ci lasciò in curiosità di sapere le sue opere"22. Sappiamo che nel 1711 servì a Vienna il neo-eletto imperatore Carlo VI ${ }^{23}$, dal 1716 lavorò a Londra al King's Theatre e, dal 1719, fu scenografo e macchinista alla Royal Academy of Music. Clerici rimase a Londra per lo meno sino al 1726. Nel 1718-1719 vi giunse anche Giovanni Bononcini come direttore della Royal Academy. In precedenza il compositore modenese aveva servito gli imperatori Leopoldo I e Giuseppe I a Vienna dal 1699 al 1711 e quindi era ben conosciuto dalla regina di Portogallo. Le polemiche tra Bononcini e il fondatore della Royal Academy lavorando quindi accanto a clerici per diversi anni Georg Friedrich Haendel sono note e nel 1733 l'italiano lasciò la capitale inglese. Così non appare casuale il fatto che nel 1734 si ritrovino a Lisbona sia Clerici che Bononcini.

La notizia dell'accresciuta attività dei teatri d'opera, sia regi che pubblici, circolava certamente tra gli artisti in cerca di ingaggio appartenenti al circuito internazionale che faceva capo ai Bibiena. La famiglia Galli Bibiena e i loro allievi costituivano, infatti, una delle più articolate reti artistiche su scala europea. Essa verteva sull'asse Bologna-Vienna. Bologna si posizionava come centro di formazione, dove Ferdinando e Francesco erano tornati in qualità di 'principi' dell'Accademia Clementina, mentre Vienna, con la presenza di Giuseppe e Antonio, rappresentava il principale polo estero e la posizione strategica privilegiata per l'irradiamento dei 'bibieneschi' verso le altre corti europee, anche quelle più distanti.

${ }^{22}$ Bologna, Biblioteca Comunale dell'Archiginnasio, Sezione manoscritti, ms B. 123, Marcello Oretti, c. 113.

${ }^{23}$ Roberto Clerici potrebbe aver fatto parte dell'entourage di Ferdinando Bibiena a Barcellona sotto Carlo III, eletto poi imperatore come Carlo VI. 
Nel 1734, a Lisbona, Roberto Clerici progettò la nuova facciata per la chiesa di Nostra Signora di Loreto, che venne costruita l'anno seguente ${ }^{24}$. L'artista clementino cercò dunque spazi sia nell'ambito del costruito che della quadratura. Dipinse infatti la sala del teatro della Trindade ${ }^{25}$, ma come architetto teatrale svolse un ruolo attivo ancora ignorato dagli studi. A lui infatti è plausibile attribuire il progetto della sala della Trinità, immaginandola come struttura lignea completa di palco attrezzato e sala con ordini di palchetti. Pur essendo un'ipotesi ancora al vaglio dei miei studi, l'architetto parmense potrebbe essere stato coinvolto anche nel progetto del Teatro da Rua do Conde, inaugurato nel 1738, e, forse, anche in quello del teatro regio di Belém inaugurato - pare - nel novembre del $1737^{26}$. In ogni caso, la presenza simultanea di Clerici e Bononcini consolidò il protagonismo degli architetti-scenografi di formazione bolognese, che, significativamente, saranno i protagonisti indiscussi della seconda metà del XVIII secolo in Portogallo.

Tra il 1739 e il 1741 Roberto Clerici si spostò a Parigi, occupando l'incarico di scenografo presso l'Academie Royale de Musique. Tra 1728 e 1737 e tra 1741 e 1744, cioè prima e immediatamente dopo la presenza di Clerici, anche il fiorentino Giovanni Niccolò Servandoni (Firenze, 1695Parigi, 1766) ricoprì lo stesso ruolo. Pur non appartenendo alla tradizione prospettico-scenografica bolognese, l'artista toscano si muoveva agilmente tra i diversi ambiti dell'archi-

${ }^{24}$ Giuseppina Raggi, Architetture dell'inganno: il lungo cammino dell'illusione, Tesi di dottorato presentata alla Università di Bologna e alla Universidade de Lisboa, 2005, vol. 1, pp. 699-700.

${ }^{25}$ Id., "Una lunga passione per l'opera in Portogallo: la regina-consorte Maria Anna d'Asburgo, l'arte dei Galli Bibiena e nuovi disegni per il Real Teatro dell'Ópera do Tejo", p. 171.

${ }^{26}$ Questo teatro potrebbe essere l'antesignano di quello poi costruito da G. C. S. Bibiena in prossimità del Palazzo di Belém, la cui esatta localizzazione è stata identificata da José Camões e Paulo Roberto Masseran e presentata durante il Colloquio Os espaços teatrais para a música na Europea do século XVIII, Queluz 30 giugno - 2 luglio 2017. 
tettura (dipinta, effimera, costruita). In giovane età plasmò il suo linguaggio sulla tradizione romana ${ }^{27}$, mentre la sua esperienza iberica si colloca tra il 1745 e il 1746. Passò prima da Madrid, decidendo poi di portarsi in Portogallo. Arrivò nell'ottobre del 1745 , in un momento in cui la malattia di Giovanni V aveva impresso un ulteriore cambio di direzione alla sua politica artistico-culturale focalizzandola quasi esclusivamente sulla patriarcale e sul nuovo complesso delle $\mathrm{Ne}$ cessidades, che prevedeva la costruzione della chiesa, palazzo e convento per gli Oratoriani. Servandoni giunse in Portogallo con molte raccomandazioni ${ }^{28}$. La buona accoglienza è confermata anche dalla rapidità con cui uscì dalla prigione quando venne incarcerato il 10 maggio 1746 a causa dello sfoggio della Croce dell'Ordine di Cristo conferitagli dal pontefice e non dal re di Portogallo. Considerando le sue alte protezioni, Servandoni potè prendere visione del progetto e del cantiere delle Necessidades. La prassi di far visionare i progetti delle opere in corso, aveva importanti precedenti come quelli di Juvarra e Albrecht inviati a Mafra. Nel 1737, infatti, il rappresentante viennese Adolph Conrad von Albrecht visitò il cantiere con l'ordine che gli venisse mostrato non solo l'edificio ma anche tutte le piante generali e parzia$\mathrm{li}^{29}$. A questa visita si deve probabilmente l'idea della pianta della biblioteca (due gallerie unite da un'ellisse centrale), simile a quella della Hofbibliothek di Vienna progettata da Fischer Von Erlach, di cui Albrecht aveva ideato il programma iconografico degli affreschi.

La relazione tra Mafra e il complesso delle Necessidades è evidente tipologicamente (chiesa, convento e palazzo). Nel 1742 Giovanni V volle trasformare la piccola cappella in

${ }^{27}$ Francesco Guidoboni, Giovanni Niccolò Servandoni (1695-1766) Architetto, Tesi di dottorato presentata alla Università La Sapienza/Université de Paris I, 2014.

${ }^{28}$ Ib., pp. 166-178.

29 Sto studiando insieme a Andrea Sommer-Mathis le lettere inviate dal conte Conrad von Albrecht custodite all'Archivio di Stato di Vienna. 
"una bellissima chiesa" ${ }^{30}$, e solo nell'anno successivo mostrò l'intento di annettervi convento e palazzo per i quali furono comprati i terreni intorno ${ }^{31}$. A mio avviso, l'ampliamento del progetto va posto in relazione all'impossibilità di recarsi a Mafra, di cui si ebbe certezza tra il 1742 e il 1743 quando il deperimento fisico del monarca si stabilizzò. L'ampliamento del progetto attivò architetti e ingegneri militari, primo fra tutti Manuel da Maia chiamato a tracciare, nell'aprile del 1745 , la pianta topografica del sito, dove non appaiono né il nartece della chiesa, sporgente rispetto alla linea di facciata del complesso, né la fontana con l'obelisco scenograficamente posta nel terrazzamento antistante affacciato sul Tago. La fontana fu completata nel giorno del compleanno del re del 1747.

Considerando la cronologia del soggiorno di Servandoni, la tempistica coincide, così come l'analisi stilistica rinvia al lessico dell'architettetto italo-francese: la serliana del nartece si ritrova in molti apparati effimeri e delle macchine di fuochi progettate dal fiorentino. Anche la fonte con l'obelisco, la quale "crea una delle poche vere prospettive urbane barocche di Lisbona" 32 , ricorre costantemente sia come elemento architettonico, sia nei suoi dipinti di paesaggi con rovine. Un disegno di Servandoni, significativamente appartenente alla collezione dell'architetto José da Costa e Silva, rappresenta un Paesaggio con rovine e obelisco egiziano ${ }^{33}$.

L'increscioso episodio della Croce dell'Ordine di Cristo determinò il rapido deterioramento della posizione di favore da Servandoni acquisita e l'intera vicenda potrebbe essere stata propiziata da alcune fazioni interne alla corte collegate

\footnotetext{
${ }^{30}$ Roma, ASV, Segr. Stato 97, c. 223, 10-07-1742.

${ }^{31}$ Manuel H. Corte-Real, O Palácio das Necessidades, Lisboa, Chaves Ferreira, 2001.

${ }^{32}$ Paulo Varela Gomes, O essencial sobre a arquitectura barroca em Portugal, Lisboa, Imprensa Nacional-Casa da Moeda, 1987.

${ }^{33}$ Guidoboni, cit., p. 170.
} 
al forte gruppo di architetti-ingegneri portoghesi cresciuti nel cantiere di Mafra. È un fatto che, dopo la scarcerazione, Servandoni si occupò dell'allestimento del teatro per gli inglesi che celebrarono la vittoria del duca di Cumberland procacciandosi l'occasione di trasferirsi a Londra nel 1746. La grande affluenza di pubblico per vedere questo teatro, in un'epoca in cui gli spettacoli profani erano stati proibiti a causa della malattia del re, rivela il fermentare di una situazione socio-politica divisa tra le spinte verso il cambiamento e il perdurare del confronto serrato tra la corte e la Santa Sede. Questo intento del monarca malato si tradusse nella decisione di costruire ex-novo la patriarcale e, più concretamente, di commissionare la cappella di São João Batista nella chiesa di San Rocco ${ }^{34}$. Gli architetti portoghesi e, in particolare, João Frederico Ludovice controllarono attentamente ogni passaggio del progetto e dell'esecuzione. Ciò non toglie che l'incarico venne affidato a architetti romani: Nicola Salvi, allievo di Canevari, e Luigi Vanvitelli. Anche la lavorazione avvenne integralmente a Roma ad opera di artefici italiani, dimostrando la persistenza del vincolo con l'architettura romana: cordone a doppia mandata che aveva attraversato l'intero regno di Giovanni V anche quando, dopo il 1732, nessun architetto italiano fu più espressamente chiamato a corte.

\section{LO SPETTACOLO DELL'ARCHITETTURA:}

i teatri Regi Di G. Carlo Sicinio Bibiena a Lisbona (1752$-1755)$

Se il serrato confronto con le arti romane si placò dopo la morte di Giovanni V, si strinse invece il legame con le specializzazioni bolognesi. L'attività dell'Istituto delle Scienze a cui era annessa l'Accademia Clementina era nota a corte sin

${ }^{34}$ António Filipe Pimentel (org.), A encomenda prodigiosa, Lisboa, Santa Casa, Dgpc, 2013; Teresa Leonor M. Vale (org), A capela de São João Batista da Igreja de São Roque, Lisboa, Santa Casa, Dgpc, 2015. 
dal 1726, quando il canonico Francesco Bianchini inviò una particolareggiata descrizione su richiesta espressa del monarca. Quando Giovanni V era ancora in vita, gli ingegneri milanesi e tedeschi chiamati per la spedizione di demarcazione dei confini in Amazzonia vennero affiancati nel 1750 dall'astronomo bolognese Giovanni Angelo Brunelli (1722-1804) e dall'accademico clementino Giuseppe Antonio Landi (1713-1791), amico e compagno di Giovanni Carlo Sicinio Bibiena.

Per quanto riguarda la passione per il teatro d'opera, sin dal primo anno di lutto stretto per la morte del sovrano, Giuseppe I prese concrete iniziative per colmare la stasi che aveva caratterizzato gli ultimi otto anni del regno del padre. Incaricò infatti il figlio di João Frederico, João Pedro Ludovice, di progettare il teatro stabile per il palazzo reale della Ribeira, chiedendo l'invio di modelli e progetti dall'Italia ${ }^{35}$. La chiamata di Giovan Carlo Sicinio Galli Bibiena (Bologna, 1717-Lisbona, 1760), che giunse a Lisbona nel marzo del 1752, rappresentò il salto di qualità capace di accelerare esponenzialmente il cambiamento. La scelta del bolognese sanciva una linea di continuità con i primi venti anni del regno precedente. Diversamente da quanto sostenuto dagli studi critici ${ }^{36}$, piuttosto che uno iato, l'arrivo dello scenografo e architetto teatrale bolognese rappresentò una ripresa di forte intensità della cultura in cui Giuseppe I era cresciuto ed era stato educato. D'altronde Maria Anna d'Asburgo era ancora in vita, esercitando una forte influenza sulla coppia reale di Giuseppe I e Mariana Vittoria di Borbone. La reginamadre vide la progettazione e la costruzione dei quattro dei cinque teatri eretti da Giovanni Carlo Sicinio Bibiena tra il 1752 e il 1755 , nei luoghi che lei stessa aveva scelto quando

${ }^{35}$ São Paulo, Biblioteca Mindlin, ms. História económico politica do reino de D. José, c. 341.

${ }^{36}$ José-Augusto França, Lisboa pombalina e o Iluminismo, Lisboa, Bertrand, 1966; Nuno Monteiro, D. José, Lisboa, Temas \& Debates, 2008. 
era sovrana di Portogallo per le rappresentazioni (i palazzi reali della Ribeira, di Belém e di Salvaterra).

Da marzo a settembre del 1752 venne realizzato, esclusivamente dall'equipe degli italiani, il teatro di corte, il cosiddetto teatro do Forte. La sala riscosse molto successo. Essa non era un ambiente improvvisato, ma un esempio della consumata capacità architettonico-teatrale dei bolognesi. Considerando la rapidità di esecuzione, la struttura fu certamente eretta in legno e adattata al salone del torrione della Ribeira che presentava una insufficiente profondità (per in palco). Venne però concepita come teatro di rappresentanza, essendovi previste logge per gli ambasciatori, assenti invece nei teatri di Salvaterra, Belém e Ajuda $^{37}$, e inaugurata nel settembre del 1752. Da marzo 1752 a febbraio 1753 venne costruito anche il teatro di Salvaterra, descritto da Giovan Angelo Brunelli in quello stesso carnevale come "un grazioso teatrino [...] dove si rappresentano commedie e opere" 38 . Tra il 1753 e il 1754 venne progettato e costruito anche il teatro regio di Belém dedicato principalmente alla commedia dell'arte ${ }^{39}$. L'ingaggio della compagnia operistica andò infatti di pari passo con l'ingaggio della compagnia di "istrioni italiani" capitanata da Antonio Sacchi, il più famoso Arlecchino attivo al tempo in Europa, seguendo il gusto introdotto dalla regina-madre sin dagli anni Venti. Infine, nel 1755, si stava costruendo il teatro regio di Ajuda, destinato alle rappresentazioni operistiche. Si stabilirono così due poli distinti ma complementari per le rappresentazioni teatrali sulle pendici della collina tra Belém e Ajuda.

La supervisione delle attività inerenti al Grande Teatro di Corte spettava a João Pedro Ludovice. La stretta collabora-

${ }^{37}$ Solo dopo gli anni Sessanta del secolo si hanno notizie di banchi in platea destinati ai rappresentanti diplomatici nei teatri di Ajuda, Queluz e Salvaterra.

${ }^{38}$ Giuseppina Raggi, "Una lunga passione per l'opera in Portogallo: la regina-consorte Maria Anna d'Asburgo, l'arte dei Galli Bibiena e nuovi disegni per il Real Teatro dell'Ópera do Tejo", p. 186 e, in particolare, n. 115, pp. 173-175, sulla diatriba inerente al Gran Teatro di Corte e bibliografia aggiornata.

${ }^{39} \mathrm{Ib}$. Vedi anche nota n. 26. 
zione con Sicinio superò quello iato tra architetti italiani e portoghesi creatosi dopo il rientro a Roma di Canevari. Giovanni Carlo Sicinio Bibiena s'inserì nelle dinamiche sociali lusitane, sposando in seconde nozze una donna portoghese e avendo come testimone proprio João Pedro Ludovice. La naturalizzazione portoghese ottenuta nel 1760 avrebbe consolidato l'unione delle due dinastie di architetti (i Bibiena e i Ludovice), se la morte non avesse colto entrambi in quello stesso anno. Li avrebbe attesi un'intensa attività architettonica, dato che mantennero la stessa relazione professionale, stabilita durante la costruzione della Real Ópera do Tejo, anche nei lavori inerenti alla cosiddetta Real Barraca.

\section{L'effimero permanente: Giovan Carlo Sicinio Bibiena e il PALAZZO REALE DI AJUdA (1756-1760)}

Il terremoto del 1755 determinò un repentino cambio di funzione appropriato alla tradizione artistica di cui Giovan Carlo era portatore ${ }^{40}$. Le compagnie ingaggiate per i teatri regi d'opera e di commedia furono smantellate. I teatri regi di Belém, Ajuda e Salvaterra continuarono attivi ma senza l'investimento economico anteriore. Tra i suoi stretti collaboratori solo Giacomo Azzolini rimase in Portogallo grazie all'invito di lavorare a Coimbra. Per quanto riguarda Sicinio, le sue capacità professionali vennero dirette all'urgenza della ricostruzione, attivando un'abilità propria della scuola bolognese. Il bisogno di creare spazi monumentali e adeguatamente decorati fece tesoro della tradizione bolognese nel campo dell'effimero, mentre l'osmosi tra effimero e costruito fu un passo imposto dalle circostanze che permise di costruire rapidamente la reale cappella nell'Ajuda così come il nucleo fondamentale della Real Barraca che, in realtà, non era affatto una baracca ${ }^{41}$.

\footnotetext{
${ }^{40}$ Per una visione generale su Bibiena vedi Jadranka Bentini; Deanna Lenzi, $I$ Bibiena. Una famiglia europea, Venezia, Marsili, 2000.

${ }^{41}$ Maria Isabel Braga Abecasis, A Real Barraca. A residência na Ajuda dos reis de Portugal após o terramoto (1756-1794), Lisboa, Tribuna da História, 2009.
} 
Le arti dell'effimero permettevano un primo e rapido impianto in legno, da cui si passava rapidamente al costruito, sostituendo al legno le murature rivestite da finti marmi, dipinti grazie alla sapienza della quadratura bolognese. Questa capacità di convertire l'effimero in architettura permanente entrò a far parte della prassi costruttiva che fronteggiava l'urgenza della ricostruzione post-terremoto. La sua applicazione è infatti riscontrabile sia nelle decorazioni interne delle chiese ricostruite della Baixa (come la chiesa di Santo António da Sé) che negli edifici regi (come la cappella del palazzo della Bemposta). La stretta collaborazione tra Giovanni Carlo Sicinio Bibiena e João Pedro Ludovice pose le premesse a favore di una maggiore integrazione degli architetti-scenografi, tra cui cominciavano a far parte anche gli allievi portoghesi del Bibiena cresciuti nel cantiere della Real Ópera do Tejo. Ciò non toglie che, in occasione delle commissioni simbolicamente più rappresentative, la corte riconoscesse all'architetto italiano maggiore capacità di garantire la magnificenza adeguata: è il caso della chiesa della Memória, progettata immediatamente dopo l'attentato al re Giuseppe I nel 1759.

In questa fase concitata di (ri)pianificazione di Lisbona operò anche l'architetto romano Giovanni Battista Antinori (Camerino, 1734-Roma, 1792), giunto a Lisbona nrl 1755 e rientrato a Roma nel $1759^{42}$. La sua attività in Portogallo attende ulteriori approfondimenti di ricerca. Il suo nome viene associato da alcune fonti al progetto per il nuovo palazzo reale nel sito di São João dos Bem Casados e a quello per un teatro pubblico previsto nell'ambito della nuova urbanizzazione della Baixa. Dopo il suo rientro a Roma fu architetto dei Doria-Pamphili e del papa Pio VI (1775-1799). A Roma mantenne relazioni privilegiate con il circuito dei

${ }^{42}$ Valeria Giontella, O arquitecto Giovanni Antinori. Vida e obra de um arquitecto que trabalhou em Lisboa e em Roma no século XVIII (relaçôes culturais e artísticas entre Lisboa e Roma no século XVIII), Tesi di dottorato presentata alla Universidade Técnica de Lisboa, 2013. 
diplomatici lusitani. Sono documentati, infatti, commissioni di apparati effimeri realizzati nella chiesa di Sant'Antonio dei Portoghesi per le celebrazioni funebri in memoria del re consorte Pedro III nel 1787 e per festeggiare l'arrivo del conte di Galveias nel 1790. Unito in matrimonio con la portoghese Josefa Luisa da Cunha, entrambi mantennero stretti rapporti con il conte, nominato ministro plenipotenziario tra la fine del 1788 e il 1790. Antinori partecipò così ai primi tentativi di organizzazione dei corsi per la formazione degli studenti della Casa Pia, inviati nel 1785 a Roma da Pina Manique ${ }^{43}$. La breve esperienza portoghese di Antinori rappresentò l'unica linea di continuità diretta con l'architettura della capitale pontificia durante la seconda metà del Settecento, dato che a partire dal 1750 gli architetti italiani che giunsero in Portogallo furono esclusivamente di origine bolognese.

\section{L'IMPRONTA BOLOGNESE NELL'ARCHITETTURA PORTOGHESE:}

Giuseppe Antonio Landi E Giacomo AzZolini (1750 - 1791)

Durante gli anni Cinquanta del secolo, prima e dopo il terremoto del 1755, la tradizione bolognese nel campo dell'architettura si consolidò anche grazie a due eventi non pianificati. Il primo riguarda il progetto di Giuseppe Antonio Landi (Bologna, 1713-Belém do Pará, 1791) per il Seminario di Coimbra e il secondo riguarda la chiamata di Giacomo Azzolini (Bologna, 1723-Lisbona, 1791) da parte del rettore Nicola Giliberti a seguito della morte di Francesco Tamossi, capo-mastro del Landi.

Nel settembre del 1766 Azzolini tornò a Lisbona, venendo messo a libro paga della Casa Real in qualità di "Architec-

\footnotetext{
${ }^{43}$ Michela Degortes "Ensino artístico no estrangeiro e relações internacionais: o caso da Academia Portuguesa de Bralas-artes em Roma”, Maria João Neto; Marize Malta (org.), Coleçôes de arte em Portugal e Brasil nos séculos XIX e XX, Lisboa, Caleidoscopio, 2016, pp. 137-148.
} 
to Pintor e Inventor das Scenas" ${ }^{34}$ dei teatri regi ${ }^{45}$. Un anno dopo, nel novembre 1767, anche il rettore del Seminario Nicola Giliberti lasciò Coimbra, andando a ricoprire il ruolo di vice-direttore del Colégio dos Nobres sino al $1772^{46}$. Nel Collegio recentemente istituito a Lisbona si creò un altro polo di diffusione della cultura scientifica e artistica bolognese, che influenzò profondamente l'architettura tra Sette e Ottocento. Nel 1765, infatti, richiamato dalla spedizione in Amazzonia, Giovanni Angelo Brunelli era stato assunto per insegnare matematica e il giovane José da Costa e Silva fu uno dei suoi allievi. Nel 1771 accompagnò l'astronomo a Bologna, aprendo un nuovo capitolo della vicenda architettonica portoghese che avrebbe inciso anche sullo sviluppo di Rio de Janeiro.

Giacomo Azzolini, invece, non rientrò più in Italia. Naturalizzato portoghese nel 1760 insieme a Giovanni Carlo Sicinio Bibiena, a Lisbona svolse un'intensa attività di scenografo per i teatri reali di Ajuda e di Salvaterra ${ }^{47}$. Il compenso di 600000 reis annui era superiore non solo a Inacio de Oliveira Bernardes, assunto a servizio regio come "Architecto Pintor" ${ }^{\prime 4}$, ma anche a quello di tutti gli altri artisti e dello stesso architetto Robillon, attivo al palazzo di Queluz, retribuito con 480000 reis annui ${ }^{49}$. La differenza di compenso

${ }^{44}$ Lisboa, Arquivo Nacional da Torre do Tombo [ANTT], Casa Real L. ${ }^{4} 463$, cc. 34-35, trascritto in Giuseppina Raggi, Architetture dell'inganno. Il lungo cammino dell'illusione, vol. 1, p. 761.

${ }^{45}$ Rui Lobo, "Gian Giacomo Azzolini (1723-1791): a Bolognese architect between Lisbon and Coimbra", Sabine Frommel; Micaela Antonnuci (coord.), Da Bologna all'Europa. Artisti bolognesi in Portogallo (XVI-XIX secolo), Bologna, Bononia University Press, 2017, pp. 203-226.

${ }^{46}$ Lisbona, Arquivo Nacional da Torre do Tombo [ANTT], Chancelaria Regia de D. José I, L. ${ }^{\circ}$ 22, cc. 123-v, trascritto in Giuseppina Raggi, Architetture dellinganno. Il lungo cammino dell'illusione, vol. 1, pp. 741-742.

${ }^{47}$ Rui Lobo, cit., con particolare distacco ai riferimenti bibliografici di Pedro Januário e Aline Gallasch-Hall de Beauvink.

${ }^{48}$ Lisbona, ANTT, Casa Real, L. ${ }^{4}$ 463, c. 37.

${ }^{49}$ Giuseppina Raggi, Architetture dell'inganno. Il lungo cammino dellillusione, vol. 1, p. 762 . 
non derivava da parzialità esterofila, ma dal fatto di attribuire esclusivamente a lui l'invenzione delle scenografie. Il riconoscimento della sua capacità d'invenzione, cioè di progettare, venne esteso certamente anche all'ambito del costruito, seguendo la stessa prassi che aveva convertito Sicinio da architetto e scenografo teatrale a progettista del primo palazzo reale di Ajuda. Anche Giacomo Azzolini transitò quindi tra scenografia, quadratura e architettura.

Nel 1783 il macchinista-architetto teatrale Petronio Mazzoni edificò la Casa da Música nel palazzo reale di Ajuda progettato nel suo primo nucleo dal Bibiena. Tutto il dispositivo continuava a seguire la lezione della scuola bolognese: materiali effimeri creavano spazi credibili. Le pareti della nuova sala ellittica furono infatti costruite in pietra, ma la cupola con lanterna, struttura ben più complessa da erigere e dispendiosa, venne realizzata in legno e poi elevata in pittura grazie all'illusione della prospettiva da Giacomo Azzolini ${ }^{50}$. L'altezza della cupola era ancora più spettacolare grazie alla scelta del punto di vista eccentrico, che recuperava la memoria delle quadrature dei capiscuola bolognesi seicenteschi come la cupola decentrata dipinta da Girolamo Curti.

Azzolini intervenne anche nel completamento della facciata e delle torri campanarie della chiesa di São Francisco de Paula. Il progetto architettonico più significativo fu quello della cavallerizza del palazzo reale di Belém, costruita tra il 1787 e il 1789 e decorata dopo la sua morte dai numerosi allievi ${ }^{51}$. Giacomo Azzolini trasmise il modus operandi proprio della tradizione bolognese e dell'Accademia Clementina. Nella rinomata istituzione l'insegnamento dell'architettura si basava sull'apprendimento delle regole della prospettiva e degli ordini secondo i trattati del Vignola. Lo studio della trattatistica cinquecentesca stava alla base della formazione

${ }^{50}$ Rui Lobo, cit., offre prova documentaria (Lisbona, ANTT, Casa Real, cx. 3129, cc. 20ss.).

${ }^{51}$ Oggi è l'antica sede del Museu Nacional dos Coches. 
nell'ambito della pittura di architettura (cioè della quadratura e della scenografia), intesa come maestra dell'architettura e non viceversa.

Confini fluidi e Confini Rigidi: José da Costa e Silva e Francesco SaVerio Fabri（TRA 1780 e 1817)

A questa tradizione appartenne José da Costa e Silva (Vila Franca de Xira, 1747- Rio de Janeiro, 1819), tanto da essere considerato dai suoi stessi connazionali un "architetto italiano" 52 . Con lui si compiva quella compenetrazione che faceva svanire i confini delle appartenenze geografiche. Accompagnando Giovanni Angelo Brunelli a Bologna, nel 1771 , si formò presso l'Accademia Clementina, viaggiando poi in Italia e conoscendo in modo particolare la città di Roma. Ritornato in Portogallo alla fine del 1779, trascorse quasi dieci anni nell'orbita di committenze italiane come, per esempio, la progettazione del presbiterio della chiesa di Nossa Senhora do Loreto. Alla fine degli anni Ottanta, con la progettazione dell'Erario Regio, iniziò a disputare con gli altri architetti portoghesi le commissioni regie. Tra il $1792 \mathrm{e}$ il 1793 costruì il teatro São Carlos, concepito come sintesi tra la memoria già mitizzata della distrutta Real Ópera do Tejo e le nuove esigenze della società lusitana. Nel 1792 la principessa-vedova Maria Francisca Benedita gli affidò il progetto del monumentale Hospital dos Inválidos Militares di Runa, la cui pianta (edificio quadrangolare con chiesa a pianta centrale in asse all'entrata) ricorda il seminario di Coimbra progettato da Landi. L'affermazione progressiva dell'architetto motivò l'inizio di una forte polemica. Da una parte, José

\footnotetext{
${ }^{52}$ Luis Soares Carneiro, Os teatros de raiz italiana em Portugal, Tesi di dottoramento presentata alla Universidade do Porto, 2003. Per la biografia artistica vedi José de Monterroso Teixeira, José da Costa e Silva (1747-1819) e a receção do Neoclassicismo em Portugal. A clivagem de discurso e a prática arquitetónica, Tesi di dottorato presentata alla Universidade Autónoma de Lisboa, 2012, 3 voll.
} 
da Costa e Silva si mostrava assai critico verso l'esperienza urbanistico-architettonica derivata dalla ricostruzione della Baixa di Lisbona ${ }^{53}$, dall'altra parte gli architetti cresciuti in questa esperienza si affermavano come legittimi rappresentanti dell'architettura detta nazionale. Nuovi concetti stavano quindi affacciandosi, e si giocavano sul filo di rivalità interne connotate come diatribe tra portoghesi e italiani. Nel 1797, infatti, l'architetto portoghese José Manuel de Carvalho e Negreiros scrisse al principe-reggente Giovanni VI un giudizio rivelatore sull'esperienza architettonica anteriore:

Fica igualmente demonstrado que os abusos praticados em Portugal, e também em algumas outras naçôes da Europa, a que os modernos chamão iluminadas [...] vem assim da liberdade com que se intitulão Architetos, e exercitão esta profissão, todo aquelle que unicamente sabe debuxar, e as cinco ordens de Vignolla assim vulgarmente chamadas; do que se segue que qualquer Entalhador, ou pintor de prospectiva se [aljao] a ser Architetos, e hé bem verdade que desenham plantas e fachadas e que muitas se executam, mas hé inegável que se deve atribuir ao accazo o bom sucesso de semelhantes obras. ${ }^{54}$

Il bersaglio della polemica era evidentemente José da Costa e Silva, in quanto accademico clementino. José Manuel de Carvalho e Negreiros era figlio di uno dei principali ingegneri e architetti militari: Eugenio dos Santos e Carvalho (1711-1760), responsabile per il disegno della ricostruzione di Lisbona e nipote per via materna di Manuel da Costa Negreiros (1702-1750), anch'egli ingegnere e architetto militare che si era formato nel cantiere di Mafra.

Negli ultimi anni del XVIII secolo la posta in gioco non era solo l'Erario Regio, ma lo stesso palazzo reale di Ajuda, dato che la cosiddetta Real Barraca era bruciata nel 1794. Il

${ }^{53}$ José da Costa e Silva propose di riposizionare il palazzo reale nell'antico Terreiro do Paço trasformato in Praça do Comércio. Vedi ib.

${ }^{54}$ Manuel H. Corte-Real, cit. 
progetto per la ricostruzione era stato assegnato a Manuel Caetano, architetto del palazzo reale di Queluz, ma Rodrigo de Sousa Coutinho, responsabile dell'Erario Regio, volle sottoporre i progetti elaborati al giudizio di José da Costa e Silva e di Francesco Saverio Fabri (Medicina, 1761-Lisbona, 1817). Fabri si era, nel frattempo, stabilito in Portogallo. Formatosi anch'egli presso l'Accademia Clementina di Bologna, aveva dapprima servito il vescovo di Faro Francisco Gomes de Avelar, venendo poi nominato Arquitecto das Obras Públicas nel $1795^{55}$. La polemica di Negreiros era dunque rivolta ad entrambi. Il nuovo palazzo reale di Ajuda rappresentava il più imponente e aulico edificio del regno. Sulla base dei loro giudizi, nel 1802 Rodrigo de Sousa Coutinho decise di accantonare il progetto di Manuel Caetano e di commissionarne di nuovi a Costa e Silva e a Fabri. Prevalse quello di José da Costa e Silva ma entrambi gli architetti lavorarono alla costruzione sino al 1812, quando Costa e Silva raggiunse la famiglia reale a Rio de Janeiro.

I tempi erano profondamente cambiati. La dialettica che aveva percorso l'intero Settecento tra scuola romana di Carlo Fontana e scuola bolognese dei Bibiena si chiudeva, all'apertura del secolo XIX, consegnando nelle mani degli architetti clementini l'edificio più rappresentativo della storia della monarchia lusitana d'Antico Regime, mentre gli accadimenti storici s'incaricarono di aggiornare la magniloquenza dell'architettura alle nuove esigenze della società portoghese... lasciandolo incompiuto.

${ }^{55}$ Giovanni Rimondini et al., Francesco Saverio Fabri: architetto (Medicina 1761Lisbona 1817). Formazione e opere in Italia e Portogallo, Medicina, Comitato Ricerche Storiche Medicinesi, 1979; Ayres de Carvalho, Os três arquitectos da Ajuda. Do "Rocaille" ao Neoclássico, Lisboa, Academia Nacional de Belas Artes, 1979; José de Monterroso Teixeira, cit. 